\title{
A versão simplificada do therapeutic intervention scoring system e seu valor prognóstico
}

\author{
THE SIMPLIFIED VERSION OF THERAPEUTIC INTERVENTION SCORING SYSTEM \\ AND ITS PROGNOSTIC VALUE
}

LA VERSIÓN SIMPLIFICADA DEL THERAPEUTIC INTERVENTION SCORING SYSTEM Y SU VALOR PRONÓSTICO

\author{
Maria Cláudia Moreira da Silva1, Regina Márcia Cardoso de Sousa²
}

\section{RESUMO}

O estudo avalia a capacidade do Therapeutic Intervention Scoring System (TISS-28) de discriminar pacientes internados em UTI, prováveis de morrer daqueles possíveis de sobreviver e estabelecer a pontuação limiar para alta probabilidade de morte. Os resultados, obtidos da amostra de 200 pacientes internados em 14 UTIs do Município de São Paulo, mostraram que o TISS-28 apresentou associação com mortalidade $(p=0,0001)$. $O$ ponto de corte estabelecido foi 21. Encontrou-se que $80,88 \%$ dos que morreram tinham pontuação do TISS-28 maior ou igual, e $68,18 \%$ dos sobreviventes tinham pontuação menor que 21 . Além disso, quanto ao valor prognóstico do TISS-28, constatou-se acurácia de 0,72.

\section{PALAVRAS-CHAVE}

Cuidados intensivos.

Prognóstico.

Índice de gravidade de doença.

\begin{abstract}
The study evaluates the competence of the "TISS-28"

to distinguish inpatients at the $I C U$, between the ones likely to die from the ones likely to survive and to establish a threshold score for high likelihood to death. The findings obtained by the sample of 200 inpatients at 14 ICUs in Sao Paulo County showed that the TISS-28 presented association with mortality ( $p=0.0001)$. The cutting score established was 21. It was found that $80.88 \%$ of those who died had the TISS-28 score similar or higher and $68.18 \%$ of survivors had the score below 21. Furthermore, regarding to the prognostic value of TISS-28, it was showed up the accuracy of 0.72 .
\end{abstract}

\section{KEYWORDS}

Intensive care.

Prognosis.

Disease severity rate.

\section{RESUMEN}

El estudio evalúa la capacidad del Therapeutic Intervention Scoring System (TISS-28) para discriminar pacientes internados en UCI, probables de morir de aquellos posibles de sobrevivir y establecer la puntuación límite para la alta probabilidad de muerte. Los resultados, obtenidos de la muestra de 200 pacientes internados en 14 UCIs del Municipio de São Paulo, mostraron que el TISS-28 presentó asociación con la mortalidad ( $p=0,0001)$. El punto de corte establecido fue 21. Se encontró que el $80.88 \%$ de los que murieron tenían puntuación del TISS-28 mayor o igual, y $68.18 \%$ de los sobrevivientes tenían puntuación menor que 21. Además de eso, en cuanto al valor pronóstico del TISS-28, se constató exactitud de 0.72 .

\section{PALABRAS CLAVE}

Cuidados intensivos.

Pronóstico.

Índice de gravedad de enfermedad.

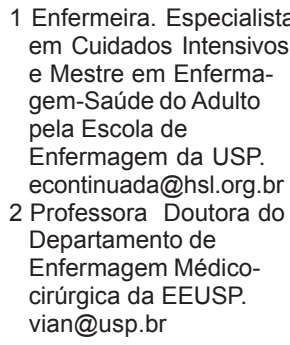

1 Enfermeira. Especialista em Cuidados Intensivos e Mestre em Enfermagem-Saúde do Adulto pela Escola de Enfermagem da USP. econtinuada@hsl.org.br

2 Professora Doutora do Departamento de Enfermagem Médicocirúrgica da EEUSP. vian@usp.br 


\section{INTRODUÇÃO}

Tem sido estimado que 15 a $20 \%$ dos pacientes americanos recebem cuidados intensivos em algum momento de sua permanência no hospital e, geralmente, a Unidade de Terapia Intensiva (UTI) é responsável por $5 \%$ do total das admissões e consome cerca de $20 \%$ do orçamento hospitalar ${ }^{(1-2)}$.

Tradicionalmente, as UTIs destinam-se ao tratamento de pacientes graves, passíveis de recuperação ou em risco de vida, o que exige que a unidade seja dotada de recursos humanos e materiais especializados para monitorização constante das funções vitais do indivíduo, a fim de prevenir e detectar possíveis complicações. Portanto, as UTIs concentram recursos humanos e tecnológicos sofisticados, geralmente não disponíveis em outras áreas do hospital, proporcionando, assim, assistência considerada como uma das mais complexas do sistema de saúde.

Alguns autores ressaltam a necessidade do desenvolvimento de métodos para predizer resultados em terapia intensiva, devido aos custos elevados e à mortalidade ${ }^{(3-4)}$.

Perante essas necessidades observou-se que, nas últimas décadas, houve o desenvolvimento de diversos escores para definir o grau de gravidade de doença e para determinar seu prognóstico. Com esse objetivo um dos índices de gravidade mais conhecidos é o Therapeutic Intervention Scoring System (TISS).

O TISS foi desenvolvido por Cullen e colaboradores pela primeira vez em 1974, parte do pressuposto de que quanto mais grave for o paciente, maior será o número de intervenções terapêuticas realizadas, independente do diagnostico ${ }^{(5)}$. Esse índice tem sofrido modificações desde então, em 1983, o TISS foi revisto e modernizado pelos seus autores, incluindo a substituição de alguns itens por outros. Permaneceu assim um escore que quantificava 76 intervenções terapêuticas e de monitorização, atribuindo pontos de um a quatro, conforme a complexidade, grau de invasividade e tempo despendido pela equipe de saúde para realizar determinado procedimento ${ }^{(6)}$.

Após a alteração para sua modernização, em 1983, o TISS foi submetido a duas modificações expressivas com a proposta de atender diferentes finalidades. Uma dessas modificações foi realizada por Cullen em 1994, e objetivava a mudança do índice para ser utilizado em unidades de cuidados intermediários ${ }^{(7)}$. A outra foi realizada por Miranda em 1996 e visava simplificar a aplicação do TISS em UTIs, alterando para 28 o número de itens a serem pontuados, sendo, então, denominado TISS-28 (Anexo A) ${ }^{(8)}$.

Um bom sistema de gravidade fornece uma estimativa acurada sobre o número de pacientes possíveis de morrer entre um grupo de pacientes similares ${ }^{(9)}$.

Para avaliar a capacidade do TISS-28 de discriminar pacientes internados em UTI, prováveis de morrer daqueles possíveis de sobreviver, e estabelecer a pontuação limiar para alta probabilidade de morte, realizou-se o presente estudo.

\section{MÉTODO}

O estudo foi realizado com dados relativos a 200 pacientes adultos internados em 14 UTIs sediadas no Município de São Paulo, sendo as unidades selecionadas através de uma amostragem casual estratificada, considerando-se os cinco Núcleos Regionais de Saúde (NRSs) do Município e a fonte mantenedora dos hospitais.

A quantidade de pacientes participantes do estudo foi estabelecida em função dos 1084 leitos de UTI ativos no Município ${ }^{(10)}$. Determinou-se o percentual de $20 \%$ que totalizou cerca de 200 participantes. Cada NRS participava com 40 pacientes na amostra.

Pacientes menores de 12 anos internados nas UTIs da amostra sorteada foram excluídos do presente estudo.

A pontuação total do TISS-28 foi obtida considerando-se um único dia de internação na unidade, escolhido aleatoriamente.

O instrumento utilizado para coleta de dados foi um formulário composto de quatro partes, onde foram registrados dados de identificação do paciente, referentes à internação na UTI, intervenções terapêuticas do TISS-28 e condição de saída do paciente da UTI.

Mediante apresentação do projeto de pesquisa aos Comitês de Ética e Pesquisa das instituições envolvidas, a coleta de dados foi autorizada. O prontuário do paciente foi utilizado como fonte primária de dados. 
A coleta de dados foi realizada em dois momentos: inicialmente procedeu-se ao registro das condições dos pacientes internados nas UTIs selecionadas e, durante um período, que variou de uma semana a 11 meses, houve retorno às referidas unidades até que se completasse a informação sobre a data de alta da UTI e o destino (alta, óbito ou transferência) dos pacientes estudados.

Os dados foram introduzidos em banco de dados e processados eletronicamente.

Inicialmente todas as variáveis foram analisadas descritivamente. Para as variáveis quantitativas esta análise foi feita por meio da observação dos valores mínimos e máximos e do cálculo de médias e desviospadrão e medianas. Para as variáveis qualitativas calculou-se frequências absolutas e relativas.

Como a hipótese de normalidade dos dados foi rejeitada utilizou-se o teste não paramétrico de Mann-Whitney na comparação das pontuações do TISS-28 entre mortos e sobreviventes.

Para obtenção do ponto de corte do TISS28 para o risco de óbito foi utilizado o modelo de regressão logistica ${ }^{(11)}$. O nível de significância utilizado para os testes foi de $5 \%$. Tendo em vista a eficiência do TISS-28 e o ponto de corte estabelecido para discriminar pacientes internados em UTI, prováveis de morrer daqueles possíveis de sobreviver, determinou-se a sensibilidade, a especificidade, o valor preditivo positivo e negativo, além da acurácia obtida na amostra ${ }^{(12)}$.

\section{RESULTADOS E DISCUSSÃO}

$\mathrm{Na}$ análise das características dos pacientes adultos internados nas UTIs do
Município de São Paulo observou-se distribuição eqüitativa dos pacientes segundo sexo e maioria de idosos $(59,36 \%)$, com predomínio daqueles com idade entre 61 e 70 anos $(32,43 \%)$. As mais freqüentes unidades de origem dos pacientes foram: Centro Cirúrgico (38,85\%), Pronto Socorro/Pronto Atendimento (22,29\%) e Unidade de Internação $(19,26 \%)$. As causas que motivaram a internação foram predominantemente os pós-operatórios imediatos, as doenças do aparelho circulatório e respiratório que estiveram presentes em 38,09\%, 32,12\% e $26,99 \%$ dos pacientes, respectivamente. Informações complemen-tares podem ser encontradas em outras publicações dos mesmos autores $^{(13-14)}$.

A média de idade dos 200 pacientes estudados foi de $56,85 \pm 17,88$ e mediana de 61 anos. A média de pontuação do TISS-28 foi de 20,14 \pm 7,06 e mediana de 20. Quanto à condição de saída do paciente da UTI, 68 (34\%) foram a óbito; destes, a média de idade foi de 58,72 $\pm 17,43$ e mediana de 62,50. Em relação à pontuação do TISS-28 desses pacientes que foram a óbito, a média foi de $24,35 \pm 5,40$ e mediana de 24 .

Estudos mostraram que a mortalidade teve boa correlação com a pontuação do TISS-28, e os escores APACHE e TISS-28 foram significantemente maiores nos pacientes que morreram quando comparados àqueles que sobreviveram durante o seguimento, concluindose, então, que o uso desses métodos de avaliação permitiria estabelecer resultado e prognóstico a curto e médio prazo $^{(15-17)}$.

Com objetivo similar aos estudos acima citados, as variáveis pontuação do TISS-28 e mortalidade durante a internação na UTI foram analisadas.(Quadro 1)
A versão simplificada do Therapeutic intervention scoring system e seu valor prognóstico

Quadro 1 - Sobrevida e óbito dos pacientes segundo pontuação do TISS-28 (São Paulo, 1999)

\begin{tabular}{|c|c|c|c|c|c|c|c|}
\hline Óbito & $\mathbf{n}^{\mathbf{o}}$ & $\begin{array}{c}\text { Média } \\
\text { (TISS-28) }\end{array}$ & $\begin{array}{c}\text { Desvio- } \\
\text { padrão } \\
\text { (TISS-28) }\end{array}$ & $\begin{array}{c}\text { Mediana } \\
\text { (TISS-28) }\end{array}$ & $\begin{array}{c}\text { Mínimo } \\
\text { (TISS-28) }\end{array}$ & $\begin{array}{c}\text { Máximo } \\
\text { (TISS-28) }\end{array}$ & $\mathbf{p}$ \\
\hline Não & 132 & 17,96 & 6,85 & 17,00 & 7,00 & 38,00 & $0,0001^{(1)}$ \\
Sim & 68 & 24,35 & 5,40 & 24,00 & 12,00 & 43,00 & \\
\hline
\end{tabular}

(1) nível descritivo de probabilidade do teste não-paramétrico de Mann-Whitney 
Maria Cláudia M. da Silva Regina Márcia C. de Sousa
O TISS-28 apresentou associação com a mortalidade $(\mathrm{p}=0,0001)$, onde os pacientes que foram a óbito apresentaram TISS-28 significativamente mais elevados do que aqueles que não foram a óbito, ou seja, à medida que aumenta a pontuação do TISS-28, aumenta também a probabilidade do indivíduo morrer. Reafirmando esse resultado, observa-se, na Figura 1, uma relação próxima a linear entre a probabilidade de óbito e a pontuação do TISS-28.

Também o grau de gravidade dos doentes quantificado pelo TISS nas primeiras 24 horas de internação foi altamente correla-cionado com a morte, sendo que não houve mortalidade na Classe I de Cullen e nas Classes II, III e IV a mortalidade foi crescente paralela à pontuação do TISS em cada uma delas. Tais classes foram categorizadas por Cullen segundo a pontuação: Classe I - 1 a 20 pontos, Classe II - 20 a 35 pontos, Classe III - 35 a 60 pontos e Classe IV - acima de 60 pontos $^{(5)}$. Sendo assim, os pacientes de cada uma das categorias tiveram um prognóstico diferente de acordo com o grau de gravidade e, além disso, a média de idade dos sobre-viventes foi significantemente menor que a dos falecidos. $\mathrm{Na}$ análise individual, não houve correlação significativa entre idade e mortalidade; além disso, os pacientes da terceira idade ( $>60$ anos) seguiam tendo um prognóstico muito aceitável; na Classe II a sobrevivência neste grupo de idade foi de $78 \%$ e na Classe III, de $48 \%$, o que contradiz a idéia de que nesse grupo não se deveriam indicar medidas avançadas de suporte.

Figura 1 - Curva estimada de probabilidade de óbito segundo a pontuação do TISS-28 ( São Paulo, 1999)

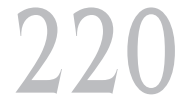

Rev Esc Enferm USP 2004; 38(2):217-24.
A fim de se obter um ponto de corte da pontuação do TISS-28 para a predição de mortalidade, utilizou-se o modelo de regressão logística, através deste modelo, foi encontrado que o ponto de corte a ser utilizado baseado nesta amostra é de 21 .

Da amostra em estudo, encontrou-se que $80,88 \%$ dos que morreram tinham pontuação do TISS-28 maior ou igual 21 , e $68,18 \%$ dos que não morreram tinham pontuação do TISS-28 menor que 21 , portanto, a sensibilidade observada nesse instrumento foi de 0,81 e a especificidade, 0,68 .
Quanto ao valor preditivo positivo, verificou-se que a probabilidade de um paciente com pontuação do TISS-28 maior ou igual 21 morrer, foi de $56,70 \%$ e o valor preditivo negativo foi de $87,38 \%$.

O "odds ratio" para a pontuação 21 do TISS-28 foi de 9,06 (intervalo de confiança a $95 \%$ : 4,47 a 18,38 ), de onde se concluiu que a chance de um paciente com TISS-28 maior que esse valor venha a morrer é 4,5 vezes maior do que aquele que apresente pontuação do TISS-28 menor que 21 . 
A capacidade discriminativa de um instrumento ou teste diagnóstico, varia de 0,5 (indicando nenhuma discriminação) a 1,0 (indicando perfeita discriminação) ${ }^{(2)}$. O valor prognóstico do TISS-28, no atual estudo, pode ser sumarizado pela acurácia de 0,72 , observada na amostra, quando estabelecido o ponto de corte de 21 . Sendo assim, pode-se afirmar que em $72 \%$ dos pacientes observados a morte e sobrevida foram corretamente prognosticadas considerando o ponto de corte estabelecido, ou seja, 21.

Autores consideram que valores maiores que 0,90 representam uma acurácia elevada e 0,70 a 0,89 representam acurácia moderada para discriminação entre sobreviventes e não sobreviventes ${ }^{(13)}$. Uma boa acurácia está próxima de $0,75^{(18)}$. Acurácia superior a 0,80 foi observada em outro trabalho, que analisou mortalidade em UTI, com a aplicação do Simplified Acute Physiology Score (SAPS II) e Acute Physiology and Chronic Health Evaluation (APACHE II ou II) nos últimos cinco anos ${ }^{19}$. Num estudo germânico, os resultados do APACHE II e TISS foram comparados, sendo observada acurácia de 0,838 e 0,798 , respectivamente ${ }^{(20)}$.

\section{CONCLUSÕES}

O valor da pontuação do TISS-28 correlacionou-se com a mortalidade no sentido em que quanto maior a pontuação, maior a chance do indivíduo morrer. Pacientes com pontuação do TISS-28 maior que 21 tiveram maior chance de morrer do que aqueles com pontuação menor que 21 , cerca de quatro vezes e meia. Entretanto, constatou-se que a acurácia desse ponto de corte do TISS- 28 foi de 0,72 . Se comparado a outros estudos que utilizaram o SAPS II e APACHE II ou III como índice de prognóstico de mortalidade, em UTI, a superioridade desses instrumentos sobre o TISS- 28 pode ser constatada.

Ainda que os resultados do presente estudo apontem para uma acurácia moderada desse instrumento para prognosticar mortalidade em indivíduos internados em UTI, maiores de 12 anos, vale aqui destacar a importância do instrumento na mensuração da carga de trabalho de enfermagem nessas unidades e sua fácil aplicação ${ }^{(14)} \mathrm{o}$ que poderá torná-lo, na prática clínica do enfermeiro, um instrumento preferido aos demais.

\section{REFERÊNCIAS}

(1) Dragsted L, Qvist J. Epidemiology of intensive care. Int J Technol Assess Health Care 1992; 8:395-407.

(2) Miranda DR, de Rijk A, Schaufeli W. Organisation and management of intensive care: a prospective study in 12 european countries. Berlin: Springer; 1998.

(3) Chen FG, Khoo ST. Critical care medicine: a review of outcome prediction in critical care. Crit Care Med 1993; 2:360-4.

(4) Mazer CD, Byrick RJ, Sibbald WJ, Chovaz PM, Goodman SJL, Girotti MJ et al. Postoperative utilization of critical care services by cardiac surgery: a multicenter study in the canadian health care system. Crit Care Med 1993; 2:851-9.

(5) Cullen DJ, Civetta JM, Briggs BA, Ferrara LC. Therapeutic Intervention Scoring System: a method for quantitative comparision of patient care. Crit Care Med 1974; 2:57-60.

(6) Keene R, Cullen DJ. Therapeutic Intervention Scoring System: update 1983. Crit Care Med 1983; 11:1-3.
(7) Cullen DJ, Nemeskal AR, Zaslavsky AM. Intermediate TISS-28: a new therapeutic intervention scoring system for non-ICU patients. Crit Care Med 1994; 22:1406-11.

(8) Miranda DR, de Rijk A, Schaufeli W.Simplified Therapeutic Intervention Scoring System: the TISS-28 items - results from a multicenter study. Crit Care Med 1996; 24:64-73.

(9) Lemeshow S, Le Gall JR. Modeling the severity of illness of ICU patients. JAMA 1994; 272:1049-55.

(10) Secretaria da Saúde do Estado de São Paulo. Apostila doc.(CD-ROM) São Paulo: 2000.

(11) Hosmer DW, Lemeshow S. Applied logistic regression. New York: John Wiley \& Sons; 1989. (Wiley series in probability and mathematical statistics).

(12) Fletcher RH, Fletcher SW, Wagner EH. Epidemiologia clínica: elementos essenciais. 3 ed. Porto Alegre: Artes Médicas; 1996.
A versão simplificada do Therapeutic intervention scoring system e seu valor prognóstico 
Maria Cláudia M. da Silva Regina Márcia C. de Sousa
(13) Silva MCM, Sousa RMC. Caracterização dos pacientes adultos e adolescentes das unidades de terapia intensiva do Município de São Paulo. Rev Paul Enferm. 2002; 21:50-9.

(14) Silva MCM, Sousa RMC. Unidades de terapia intensiva do Município de São Paulo: diferenças entre pacientes dos Núcleos Regionais de Saúde e dos hospitais governamentais e não governamentais. Rev Bras Ter Int 2001; 13(1):6-14.

(15) Artucio H, Garrido R, Buccino J, Poggi C, Fraschini R. Utilizacion de los indices APACHE y TISS-28 en una unidad de medicina intensiva. Paciente Crit 1988; 1:142-58.

(16) Parada MT, Barros L, Wainstein E, Mascaró J, Soto L. Aplicacion de dos sistemas de evaluacion, APACHE y TISS-28, en pacientes bronquiticos cronicos ingressados a una unidad de cuidados intensivos. Enferm Respir Cir Torac 1988; 4:15-7.
(17) Reyes J, D’Empaire G. Indices de severidad y prognostico en una unidad de medicina critica privada. Med Crit Venez 1992; 7:11-22.

(18) Auriant I, Vinatier I, Thaler F, Tourneur M, Loirat P. Simplified Acute Physiology Score II for measuring severity of illness in intermediate care units. Crit Care Med 1998; 26:1368-71.

(19) Capuzzo M, Valpondi V, Sgarbi A, Bortolazzi S, Pavoni V, Gilli et al. Validation of severity scoring systems SAPS II and APACHE II in a single center population. Intensive Care Med 2000; 26: 1779-85.

(20) Von Bierbrauer A, Burchardt C, Muller HH, Von Wichert P. Value of the Hannover Intensive Score (HIS) in internal medicine intensive care. Med Klin 1998; 93:524-32. 


\section{ANEXO A \\ THERAPEUTIC INTERVENTION SCORING SYSTEM (TISS-28)}

-Monitorização padrão. Sinais vitais horários, registros e cálculo regular do balanço hídrico.

-Laboratório. Investigações bioquímicas e microbiológicas.

-Medicação única. Endovenosa, intramuscular, subcutânea, e/ou oral (por exemplo, sonda nasogástrica)

-Medicações endovenosas múltiplas. Mais do que uma droga. Injeções únicas ou contínuas.

- Troca de curativos de rotina. Cuidado e prevenção de úlceras de decúbito e troca diária de curativo.

-Trocas frequentes de curativos. Troca frequente de curativo (pelo menos uma vez por turno de enfermagem) e/ou cuidados com feridas extensas.

\section{Suporte Ventilatório}

-Ventilação mecânica. Qualquer forma de ventilação mecânica/ventilação assistida com ou sem pressão expiratória positiva final, com ou sem relaxantes musculares; respiração espontânea com pressão expiratória positiva final.

-Suporte ventilatório suplementar. Respiração espontânea através do tubo endotraqueal sem pressão expiratória positiva final; oxigênio suplementar por qualquer método, exceto aplicação de parâmetros de ventilação mecânica.

-Tratamento para melhora da função pulmonar. Fisioterapia torácica, espirometria estimulada, terapia de inalação, aspiração endotraqueal.

\section{Suporte Cardiovascular}

-Medicação vasoativa única. Qualquer droga vasoativa

-Medicação vasoativa múltipla. Mais de uma droga vasoativa, independente do tipo e dose.

-Reposição endovenosa de grandes perdas volêmicas. Administração de volume $>31 / \mathrm{m}^{2} / \mathrm{dia}$, independente do tipo de fluido administrado. 
Pontuação

-Monitorização do átrio esquerdo. Cateter de artéria pulmonar com ou sem medida de débito cardíaco...

-Via venosa central...

-Ressuscitação cardiopulmonar após parada cardiocirculatória nas últimas 24 horas (soco precordial único não incluído).

. .3

\section{Suporte Renal}

-Técnicas de hemofiltração. Técnicas dialíticas 3

-Medida quantitativa do débito urinário (por exemplo, sonda vesical de demora) 2

-Diurese ativa (por exemplo, Furosemide $>0,5 \mathrm{mg} / \mathrm{Kg} /$ dia por hipervolemia). 3

\section{Suporte Neurológico}

-Medida de pressão intracraniana. .4

\section{Suporte Metabólico}

-Tratamento da acidose/alcalose metabólica complicada .4

-NPT - Nutrição Parenteral Total endovenosa. 3

-Nutrição enteral através da sonda nasogástrica ou outra via gastrointestinal (por exemplo, jejunostomia)

\section{Intervenções Específicas}

-Intervenção específica única na UTI. Intubação naso ou orotraqueal, introdução de marca-passo, cardioversão, endoscopia, cirurgia de emergência nas últimas 24 horas, lavagem gástrica: não estão incluídas intervenções de rotina sem consequências diretas para as condições clínicas do paciente, tais como radiografias, ecografias, eletrocardiograma, curativos, introdução de cateter venoso ou arterial.

-Intervenções específicas múltiplas na UTI. Mais do que uma conforme descritas acima.

-Intervenções específicas fora da UTI. Procedimentos diagnósticos ou cirúrgicos.

"Medicação endovenosa múltipla" exclui "medicação única",

"ventilação mecânica" exclui "suporte ventilatório suplementar",

"medicação vasoativa múltipla" exclui "medicação vasoativa única" e

"intervenções específicas múltiplas na UTI" exclui "intervenção única na UTI".

TOTAL: pontos 